Meta

Journal des traducteurs

Translators' Journal

\title{
Liste des évaluateurs et des conseillers spéciaux 2008
}

Volume 53, numéro 4, décembre 2008

URI : https://id.erudit.org/iderudit/044184ar

DOI : https://doi.org/10.7202/044184ar

Aller au sommaire du numéro

Éditeur(s)

Les Presses de l'Université de Montréal

ISSN

0026-0452 (imprimé)

1492-1421 (numérique)

Découvrir la revue

Citer ce document

(2008). Liste des évaluateurs et des conseillers spéciaux 2008. Meta, 53(4),

729-730. https://doi.org/10.7202/044184ar d'utilisation que vous pouvez consulter en ligne.

https://apropos.erudit.org/fr/usagers/politique-dutilisation/ 


\section{Liste des évaluateurs et des conseillers spéciaux 2008}

Nous remercions chaleureusement tous ceux et celles qui ont donné de leur temps et qui contribué à la revue. Toute omission est involontaire de notre part, veuillez nous la signaler.

Beverly Adab (Aston University)

Barbara Ahrens (Cologne University of Applied Sciences)

Bassey E. Antia (University of Maiduguri)

Louise Audet (Université de Montréal)

Fabienne Baider (University of Cyprus)

Christian Balliu (ISTI, Bruxelles)

Paul Bandia (Concordia University)

Salah Basalamah (University of Ottawa)

Nicole Baumgarten (University of Southern Denmark)

Henri Béjoint (Université Lumière-Lyon II)

Daniel Blampain (Bruxelles)

Annie Brisset (University of Ottawa)

Louise Brunette (Université du Québec en Outaouais)

Deborah Cao (Griffith University)

Andrew Chesterman (University of Helsinki)

Jungwha Choi (Université Hankuk des Études Étrangères)

Mikyung Choi (Université Féminine d'Ewha)

Andrew Clifford (York University)

Katleen Connors (Université de Montréal)

Monique C. Cormier (Université de Montréal)

Jeanne Dancette (Université de Montréal)

Jean Delisle (Université d'Ottawa)

Jorge Díaz-Cintas (Imperial College London)

Joseph Dichy (Université Lumière-Lyon II)

Pascaline Dury (Université Lumière-Lyon II)

Álvaro Echeverri (Université de Montréal)

Mohammed Farghal (Yarmouk University)

Martin Forstner (Universität Mainz)

Ryan Fraser (University of Ottawa)

Judit Freixa (Université Pompeu Fabra)

Yves Gambier (Université de Turku)

Daniel Gile (ESIT, Paris)

Jean-Claude Gémar (Université de Montréal)

Roger Goffin (Université Libre de Bruxelles)

Dinda L. Gorlée (University of Bergen)

Daniel Gouadec (Université de Haute Bretagne - Rennes 2)

Frédéric Grognier (Université de Moncton)

Mathieu Guidère (Université de Genève)

Tony Hartley (University of Leeds)

John Humbley (Université Paris Diderot - Paris VII)

Amparo Hurtado Albir (Universitat Autònoma de Barcelona)

Moira Inghilleri (University College London) 
Klaus Kaindl (University of Vienna)

Dorothy Kelly (Universidad de Granada)

Hyerim Kim (Université Féminine d'Ewha)

Aurelia Klimkiewicz (York University)

Jane Koustas (Brock University)

Louise Ladouceur (University of Alberta)

Francisco Lafarga (Université de Barcelone)

Sylvie Lambert (University of Ottawa)

Laurent Lamy (Université de Montréal)

Christopher Larkosh (University of Massachusetts Dartmouth)

Hannelore Lee-Jahnke (ÉTI, Genève)

$\mathrm{Mu}$ Lei (Hainan University)

Defeng Li (University of London)

Kazem Lotfipour-Saedi (University of Tabriz)

Serge Marcoux (Université de Montréal)

Elizabeth Marshman (University of Ottawa)

Raymond Mopoho (Dalhousie University)

Christiane Nord (University of Applied Sciences of Magdeburg)

Alexis Nouss (Université de Montréal)

Pilar Orero (Universitat Autònoma de Barcelona)

Éliane Orléans-Gerstein (Montréal)

Man-Ghyu Pak (Ajou University)

Mayra Parra (Université de Montréal)

Franz Pöchhacker (University of Vienna)

Brian Rainey (Regina University)

Sathya Rao (University of Alberta)

Jen Rasmussen (Copenhague)

Roda Roberts (Université d'Ottawa)

André Roman (Université Lumière - Lyon II)

Antin Fougner Rydning (Université d'Oslo)

Julio César Santoyo (Universidad de León)

Véronique Sauron (Université de Genève, ETI)

Wei Shao (Université des langues étrangères de Beijing)

Miriam Shlesinger (Bar-Ilan University)

Candace Séguinot (York University)

Berndt Stefanink (Université de Bielefeld)

Paul St-Pierre (Université de Montréal)

Sehnaz Tahir-Gürçağlar (Bogazici University)

Kayoko Takeda (Monterey Institute of International Studies)

Claude Tatilon (York University)

John Thorp (University of Western Ontario)

Rita Temmerman (Erasmushogeschool Brussel)

Louis Truffaut (Genève)

Egan Valentine (Université du Québec à Trois-Rivières)

Luise von Flotow (University of Ottawa)

Wolfram Wilss (Universität des Saarlandes)

Jun Xu (Université de Nanjing)

Yong Zhong (University of New South Wales)

Shodja Ziaian (York University) 